\title{
Spatial Stability of Incompressible Attachment-Line Flow
}

\author{
Vassilios Theofilis \\ Department of Applied Mathematics, University of Twente, \\ P.O. Box 217, 7500 AE Enschede, The Netherlands
}

\begin{abstract}
Linear stability analysis of incompressible attachment-line flow is presented within the spatial framework. The system of perturbation equations is solved using spectral collocation. This system has been solved in the past using the temporal approach and the current results are shown to be in excellent agreement with neutral temporal calculations. Results amenable to direct comparison with experiments are then presented for the case of zero suction.

The global solution method utilized for solving the eigenproblem yields, aside from the well-understood primary mode, the full spectrum of least-damped waves. Of those, a new mode, well separated from the continuous spectrum is singled out and discussed. Further, relaxation of the condition of decaying perturbations in the far-field results in the appearance of sinusoidal modes akin to those found in the classical Orr-Sommerfeld problem.

Finally, the continuous spectrum is demonstrated to be amenable to asymptotic analysis. Expressions are derived for the location, in parameter space, of the continuous spectrum, as well as for the limiting cases of practical interest. In the large Reynolds number limit the continuous spectrum is demonstrated to be identical to that of the Orr-Sommerfeld equation.
\end{abstract}

\section{Introduction}

It has long been established that linear theory may be utilized to describe the instability of smallamplitude perturbation waves in the incompressible attachment-line boundary layer. Hall et al. (1984) employed analysis and a local temporal model which efficiently yields the exact location, in parameter space, of the neutral loop pertinent to this problem. Information obtained through this temporal approach on the frequency and growth rate of the most dangerous, from a stability point of view, instability mode may be related to that observed experimentally by means of the approximate relation between temporal and spatial results provided by the Gaster transformation (Gaster, 1962). Partly due to the computing cost of the spatial approach (a function of the numerical method that has been utilized) and partly because spatial results may be inferred from the temporal ones, stability work following that of Hall et al. has either utilized the temporal model or employed a local approach to obtain spatial results (see Malik and Beckwith, 1988).

If the linear critical Reynolds number is the result sought, the temporal analysis reported by Hall et al. suffices. The much more expensive spatial analysis is warranted, however, on the grounds of the Gaster transformation being very accurate near the neutral loop only. When the growth rate of a particular unstable wave becomes large enough, away from the neutral loop, Gaster's transformation may still be used in an approximate sense; experimental results in this case, though, are best compared with those yielded by spatial analysis. 
Further motivation for a spatial linear stability analysis is provided by the need for validation of Direct Numerical Simulation (DNS) results. DNS should supply information on the aspects of an instability problem that linear analysis does not take into account, namely, three-dimensional nonlinear interaction of instability modes. The attachment-line problem has been identified as being a prime candidate for application of a spatial DNS (Spalart, 1988). A stringent test that DNS results should be subjected to is comparison of the growth rates and eigenfunction structure obtained by DNS with results of linear theory. If the amplitude of perturbations introduced into the numerical solution of the full Navier-Stokes is kept low, DNS results should match those of linear theory to within a very low tolerance (Kleiser and Zang, 1991).

In this paper we address the global problem of spatial instability of viscous incompressible flow over an infinite swept attachment-line boundary layer, at the attachment line itself (the interested reader will find a discussion of the inviscid spatial stability problem at and off the attachment line in Hall and Seddougui (1990)). We demonstrate the application of a solution method akin to that presented by Bridges and Morris (1984) for the solution of the Orr-Sommerfeld equation. The global nature of the present approach permits the recovery of the full spectrum of eigenmodes. Amongst them, aside from the unstable mode whose temporal equivalent is discussed by Hall et al., a new (damped) mode, similar to that observed in Orr-Sommerfeld studies (Bridges and Morris, 1984), is discovered.

After a short introduction, we derive, in Section 3 the conditions that the growth rates must satisfy in order for the respective eigenfunctions to decay algebraically or exponentially in the far-field (Hall et al., 1984). These conditions are Reynolds number dependent; taking the limit of large Reynolds number we demonstrate that the continuous spectrum of the incompressible attachment-line problem merges into that of the classical Orr-Sommerfeld equation. In Section 4 the numerical solution method is presented and applied to the system governing the behavior of small-amplitude instability waves. Results are obtained in Section 5 and compared with the temporal results of Hall et al. (1984) at neutral conditions; excellent agreement is demonstrated. Further, the dependence of growth rate on frequency is utilized in order to present the frequencies which maximize (spatial) growth. The continuous spectrum is discussed next, in particular its least-stable member. It is, finally, demonstrated that the attachment-line system, in a similar manner to the Orr-Sommerfeld problem, supports eigensolutions which do not merge into the freestream value of undisturbed velocity but, rather, exhibit oscillatory (sinusoidal) behavior to infinity (Grosch and Salwen, 1978). Concluding remarks are offered in Section 6.

\section{The Physical Problem}

The introduction to the physical problem of the attachment line is presented comprehensively in a number of sources (Poll, 1979; Hall et al., 1984) and is omitted here. The flow is schematically depicted in Figure 1. A spanwise component is added to the classical stagnation (Hiemenz, 1911) incompressible flow resulting in the formation of a strictly parallel boundary layer at the attachment line itself. Invoking the Görtler-Hämmerlin (Görtler, 1955; Hämmerlin, 1955) assumption Hall et al. (1984) demonstrated that a temporal linear stability analysis of the problem yields results that compare very well with experiments, insofar as the critical Reynolds number is concerned. Hall et al. also assessed the influence of suction or blowing at the wall on this critical Reynolds number. Theofilis (1993) presented a formulation of the governing equations as an initial value problem and demonstrated that equivalent results may be obtained with the eigenvalue problem considered by Hall et al., again within a temporal framework.

For completeness the system of equations to be solved is presented first. This system results from substitution of small-amplitude perturbations into the full (incompressible) Navier-Stokes equations and subtraction of the base field. This base flow is an exact solution to the Navier-Stokes equations and, as such, stability analysis may be performed in a self-consistent manner; the generalized Hiemenz flow is discussed, for example, by Rosenhead (1963) and Schlichting (1979). The smallness of the perturbation wave amplitudes permits linearization about the base flow and a system of equations results for the determination of the perturbation velocity $\tilde{u}$, the component in the streamwise direction (normal to the attachment line, on the plane of the cylinder), and $\tilde{v}$, the normal perturbation velocity 
Figure 1. Schematic representation of the attachment-line boundary layer.

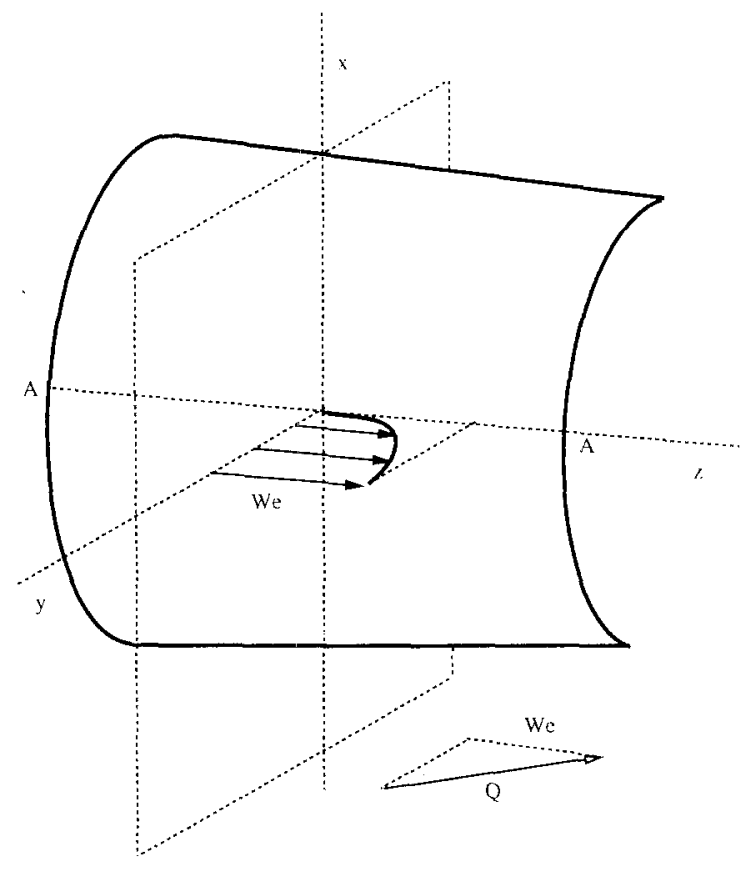

component. Continuity may be used to calculate the spanwise velocity component $\tilde{w}$, along the attachment line, once the other two velocity components are known. Pressure may be eliminated from the governing equations; this is advantageous computationally since the absence of pressure does away with the additional requirement for a staggered grid in the solution approach. The system to be solved for $\tilde{u}$ and $\tilde{v}$ is (Hall et al., 1984)

$$
\begin{gathered}
\left\{\mathscr{D}^{2}-\bar{v} \mathscr{D}-\beta^{2}-2 \bar{u}-\mathrm{i} \beta R \bar{w}\right\} \tilde{u}-(\mathscr{D} \bar{u}) \tilde{v}+\mathrm{i} \omega R \tilde{u}=0 \\
\{2(\mathscr{D} \bar{u})+2 \bar{u} \mathscr{D}\} \tilde{u}+\left\{\mathscr{D}^{4}-\bar{v} \mathscr{D}^{3}+\left[-2 \beta^{2}-\mathrm{i} \beta R \bar{w}-(\mathscr{D} \bar{v})\right] \mathscr{D}^{2}+\left[\beta^{2} \bar{v}+(\mathscr{D} \bar{u})\right] \mathscr{D}\right. \\
\\
\left.\quad+\beta^{4}+\mathrm{i} \beta^{3} R \bar{w}+\mathrm{i} \beta R\left(\mathscr{D}^{2} \bar{w}\right)+\beta^{2}(\mathscr{D} \bar{v})+\left(\mathscr{D}^{2} \bar{u}\right)\right\} \tilde{v}+\mathrm{i} R \omega\left\{\mathscr{D}^{2}-\beta^{2}\right\} \tilde{v} \\
=0,
\end{gathered}
$$

where $\mathscr{D}=d / d \eta$, with $\eta$ the normal coordinate scaled on the boundary-layer length scale $\Delta .{ }^{1}$ The base (Hiemenz) flow velocity components are $(\bar{u}, \bar{v}, \bar{w})$ and $R$ is the Reynolds number of the flow (customarily denoted by $\bar{R}$ ); a plane wave form has been assumed for all perturbation quantities, typically $\tilde{u}(\eta) e^{\mathrm{i}(\beta z-\omega t)}$, with $z$ denoting the spanwise coordinate and $t$ time.

The physical significance of $\beta$ and $\omega$ depends on the stability problem to be addressed; thus far, no statement has been made regarding whether one chooses to solve the temporal ( $\beta$ real, $\omega$ complex) or spatial ( $\beta$ complex, $\omega$ real) problem. Indeed, Hall et al. (1984) solved the temporal problem and obtained physical information of interest, such as frequency of least-stable waves, as part of the solution. However, as has already been mentioned, it is of interest to solve the spatial problem directly, in advance prescribing the frequencies of interest $(\omega$ real) and monitoring the response of the layer (spatial growth rate, $\beta_{\mathrm{i}} \equiv \mathfrak{I}\{\beta\}$ ); as part of the solution of this spatial problem the wave number of the instability wave $\beta_{\mathrm{r}} \equiv \mathfrak{R}\{\beta\}$ may be obtained.

The boundary conditions associated with the system to be solved deserve some discussion. The base-flow freestream behavior was derived by Hall et al. (1984); it was shown that $\bar{v} \sim-\left(\eta-\delta^{*}\right)$, with $\delta^{*}$ the boundary-layer thickness, while the governing equations for the base flow may be used to derive the asymptotic behavior of the streamwise $\bar{u}$ and spanwise $\bar{w}$ velocity components. On the perturbation quantities at the wall, $\eta=0$, the viscous condition of zero perturbations is imposed on both perturbation velocity components, alongside zero derivative for the normal perturbation velocity.

\footnotetext{
${ }^{1} \Delta$ is usually defined with the aid of the local strain rate of the flow, in view of the absence of a physical length scale of the problem; here, the definition of Hall et al. is adopted, although other options are also available (Hall and Malik, 1986).
} 
In the far-field, vanishing of perturbations at a sufficiently large distance from the wall may be imposed or the asymptotic form of the equations in this limit may be utilized. Hall et al. (1984) demonstrated that the proper rate of decay for the perturbations in the far-field is imposed if

$$
(\eta \rightarrow \infty) \quad \tilde{u} \sim e^{-\eta^{2} / 2}, \quad \tilde{v} \sim e^{-\beta \eta} .
$$

The latter condition is, of course, typical of incompressible Navier-Stokes stability calculations, in that the streamwise and normal perturbation velocity components acquire the usual disparity between the vortical and the irrotational part of the flow.

Imposition of a prespecified rate of decay at infinity is necessary, from a numerical point of view, in order to shorten the integration range and, thus, minimize the computing cost. However, it may be restrictive in that it excludes admissible solutions of (1)-(2) other than those prescribed by the asymptotic behavior. In an attempt to avoid introducing this a priori in the solution, we impose the former condition, namely, vanishing of perturbations, alongside with zero derivative on the normal perturbation velocity component at both endpoints of the integration domain. In the numerical results obtained, we demonstrate that the asymptotic behavior used by Hall et al. does indeed yield the physically most interesting least-stable mode; however, it does preclude other (more stable and, potentially, less interesting physically) eigensolutions supported by (1)-(2) from manifesting themselves in the full spectrum.

\section{The Continuous Spectrum}

As part of the global solution method utilized, the full spectrum of eigensolutions may be obtained. If the eigenvectors obtained are to merge into the undisturbed field, in the limit of large $\eta$, solutions exhibiting algebraic or exponential decay are sought. ${ }^{2}$ This results in solutions which behave like ${ }^{3}$

$$
\tilde{u} \sim\left(\eta-\delta^{*}\right)^{Q_{m}}, \quad \tilde{v} \sim\left(\eta-\delta^{*}\right)^{Q_{n}}, \quad m, n=1, \ldots, 5,
$$

with the admissible exponents assuming the values

$$
\begin{aligned}
& Q_{1}=-\mathrm{i} \omega R+2+\mathrm{i} \beta R+\beta^{2}, \\
& Q_{2}=-\mathrm{i} \omega R+1+\mathrm{i} \beta R+\beta^{2}, \\
& Q_{3}=-\mathrm{i} \omega R-1+\mathrm{i} \beta R+\beta^{2}, \\
& Q_{4}=+\mathrm{i} \omega R-2-\mathrm{i} \beta R-\beta^{2}, \\
& Q_{5}=+\mathrm{i} \omega R-3-\mathrm{i} \beta R-\beta^{2} .
\end{aligned}
$$

The corresponding solutions are identical to those presented in the temporal work of Hall et al. (1984). Focusing on the spatial problem, damped solutions are ensured if

$$
\mathfrak{R}\left\{Q_{n}\right\}<0, \quad \mathfrak{J}\left\{Q_{n}\right\}=0, \quad n=1, \ldots, 5 .
$$

The second condition determines the growth rate, $\beta_{\mathrm{i}}$, always given by

$$
\beta_{\mathrm{i}}=\frac{1}{2} R\left(\frac{\omega}{\beta_{\mathrm{r}}}-1\right) \text {. }
$$

The first condition results in the following expressions for the real part $\beta_{\mathrm{r}}$ of $\beta$ :

$$
\left\{\beta_{\mathrm{r}}\right\}_{n}=\left\{\frac{1}{2}\left[\left(\lambda_{n}^{2}+R^{2} \omega^{2}\right)^{1 / 2}-\lambda_{n}\right]\right\}^{1 / 2}, \quad n=1, \ldots, 5,
$$

where

$$
\lambda_{n}= \begin{cases}\frac{1}{4} R^{2}\left(1+\kappa^{2}\right)+\mu_{n}, & n=1,2,3, \\ \frac{1}{4} R^{2}\left(1-\kappa^{2}\right)+\mu_{n}, & n=4,5,\end{cases}
$$

\footnotetext{
${ }^{2}$ The condition of decay may be relaxed by imposing boundedness on the solution (Grosch and Salwen, 1978); this possibility will be discussed in the sequel.

${ }^{3}$ The author wishes to acknowledge the comment of a referee regarding the admissible combinations of exponents $Q_{m, n}$. While the interested reader may find full discussion on the matter in Hall et al. (1984), it suffices here to stress that $m \neq n$.
} 
with $\kappa$ a nonzero real number, $\mu_{1}=2, \mu_{2}=1, \mu_{3}=-1, \mu_{4}=2$, and $\mu_{5}=3$. In a typical boundary layer the condition

$$
\frac{\omega}{R} \ll 1
$$

holds, implying

$$
\left\{\beta_{\mathrm{r}}\right\}_{n} \approx \begin{cases}\frac{\omega\left[\left(1+\kappa^{2}\right)+4 \mu / R^{2}\right]^{3 / 2}}{\left[\left(1+\kappa^{2}\right)+4 \mu / R^{2}\right]^{2}+2(\omega / R)^{2}}, & n=1,2,3, \\ \frac{\omega\left[\left(1-\kappa^{2}\right)+4 \mu / R^{2}\right]^{3 / 2}}{\left[\left(1-\kappa^{2}\right)+4 \mu / R^{2}\right]^{2}+2(\omega / R)^{2}}, & n=4,5,\end{cases}
$$

the approximate value of the growth rate being derived from (8). While the approximate forms for $\beta_{\mathrm{r}}$ derived from the exponentially decaying solutions are restrictive, the algebraically decaying forms $Q_{1}$, $Q_{2}$, and $Q_{3}$ permit an arbitrary value of $\kappa$. In what follows we, therefore, focus on the algebraic solutions. Up to this point the asymptotic results derived under condition (10) are Reynolds number dependent. If, further, largeness of the Reynolds number is assumed, the following limiting cases of interest result (dropping the index $n$ ):

$$
\begin{gathered}
(\kappa \rightarrow 0) \quad \beta_{\mathrm{r}} \rightarrow \omega, \quad \beta_{\mathrm{i}} \rightarrow R\left\{\frac{1}{4} \kappa^{2}+\left(\frac{\omega}{R}\right)^{2}\right\}, \\
(\kappa \rightarrow \infty) \quad \beta_{\mathrm{r}} \rightarrow \frac{\omega}{\kappa}, \quad \beta_{\mathrm{i}} \rightarrow \frac{1}{2} R \kappa .
\end{gathered}
$$

It is straightforward to calculate the analytic dependence of $\beta_{\mathrm{i}}$ on $\beta_{\mathrm{r}}$ in the limits considered. For instance, with $\kappa \rightarrow 0$ the two solutions (stemming from the quadratic dependence of $\beta_{\mathrm{r}}$ on $\omega$ ) are

$$
\begin{aligned}
& -\left\{\beta_{\mathrm{i}}\right\}_{1}=-\frac{\sqrt{R^{2}-8 \beta_{\mathrm{r}}^{2}}}{4}-R \ln \left(\beta_{\mathrm{r}}\right)-\frac{3 R \ln (2)}{4}+\frac{R \ln \left(R+\sqrt{\left.R^{2}-8 \beta_{\mathrm{r}}^{2}\right)}\right.}{4}, \\
& -\left\{\beta_{\mathrm{i}}\right\}_{2}=\frac{\sqrt{R^{2}-8 \beta_{\mathrm{r}}^{2}}}{4}-\frac{R \ln \left(\beta_{\mathrm{r}}\right)}{2}-\frac{R \ln \left(R+\sqrt{\left.R^{2}-8 \beta_{\mathrm{r}}^{2}\right)}\right.}{4} .
\end{aligned}
$$

A noteworthy observation derived from the limiting values presented is that in all cases the growth rates are positive, indicating (massively) stabilized modes. Further, the large $R$ result for the limiting cases $\kappa \rightarrow 0$ and $\kappa \rightarrow \infty$ is, in fact, identical to that obtained in the classical Orr-Sommerfeld problem (Grosch and Salwen, 1978). This comes against a background of a rather different structure of the governing equations, namely, the existence, in the attachment-line problem, of a base-flow component (the normal velocity $\bar{v}$ ) linearly dependent on the normal coordinate, as opposed to a base flow which approaches a constant in the far-field in the Orr-Sommerfeld problem. Significantly, though, in attachment-line flow largeness of Reynolds number has to be invoked for the limiting cases to compare with the Orr-Sommerfeld problem. The present analysis, although not dealing with the fundamental mode, points out the similarities in the spectra of the attachment-line problem (1)-(2) and the nonrationally obtained Orr-Sommerfeld equation for the same problem (Poll, 1978; Arnal, 1992), paving the way for an explanation of the observed asymptotic agreement of the respective results.

\section{Numerical Solution of the Spatial Stability Problem}

Turning to the computation of the spatial linear stability problem, we first outline the solution approach. The numerical method consists of writing (1)-(2) as a nonlinear system, in symbolic form,

$$
\mathbf{A}_{i} \varphi=\sum_{i=1}^{4} \beta^{i} \mathbf{B}_{i} \varphi
$$

with $\beta$ the (complex) eigenvalue and $\mathbf{A}$ and $\mathbf{B}$ arrays whose entries are presented shortly. Equation (11) is then discretized using spectral collocation based on Chebyshev polynomials. Herbert (1977) and 

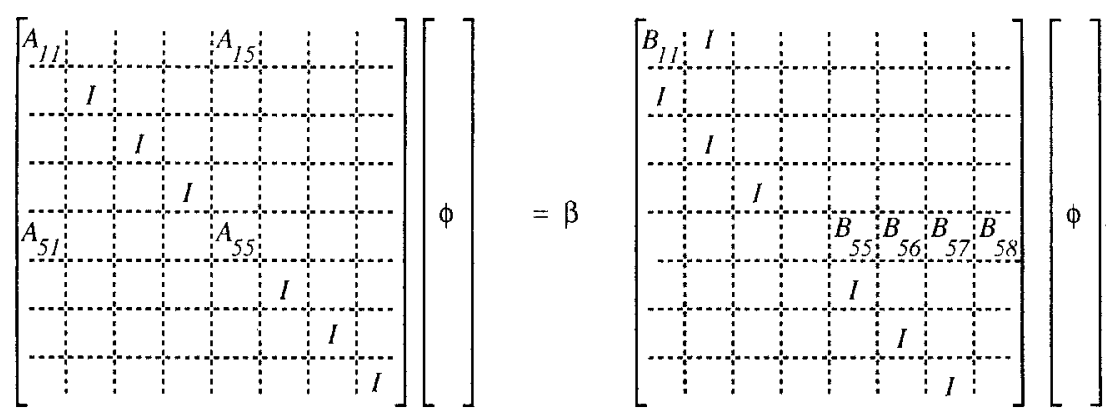

Figure 2. Setup for the numerical solution of the spatial problem; only nonzero matrices entries are shown.

Macaraeg et al. (1988) reported the earliest applications of spectral collocation for the solution, respectively, of incompressible and compressible stability eigenvalue problems. More recently Malik (1990) and Thumm (1991) reported similar results. Denoting the vector of unknowns with

$$
\varphi=\left[\tilde{u}, \beta \tilde{u}, \beta^{2} \tilde{u}, \beta^{3} \tilde{u}, \tilde{v}, \beta \tilde{v}, \beta^{2} \tilde{v}, \beta^{3} \tilde{v}\right]^{T}
$$

we set the problem up as a large linear eigenvalue problem, schematically depicted in Figure 2.

Only the nonzero entries are shown in Figure 2, where $I$ represents the identity matrix and the matrices $A_{i j}$ and $B_{i j}$ are the discrete analogues of terms in (1)-(2) built with the aid of the collocation derivative matrices $D^{(m)}$ of the Chebyshev polynomials (Gottlieb et al., 1984). A mapping has been utilized transforming the calculation grid $\eta \in\left[0, \eta_{\max }\right]$ onto the standard Chebyshev $x \in[-1,1]$. If $\hat{D}=(d x / d \eta) D$, the entries of the left- and right-hand side arrays are

$$
\begin{aligned}
& A_{11}=\hat{D}^{(2)}-\bar{v} \hat{D}-2 \bar{u}+\mathrm{i} \omega R, \\
& A_{15}=-\hat{D} \bar{u}, \\
& A_{51}=2 \bar{u} \hat{D}+2(\hat{D} \bar{u}), \\
& A_{55}=\hat{D}^{(4)}-\bar{v} \hat{D}^{(3)}+[\mathrm{i} \omega R-(\hat{D} \bar{v})] \hat{D}^{(2)}+(\hat{D} \bar{u}) \hat{D}+\left(\hat{D}^{(2)} \bar{u}\right), \\
& B_{11}=\mathrm{i} R \bar{w} \\
& B_{55}=\mathrm{i} R\left[\bar{w} \hat{D}^{(2)}-\left(\hat{D}^{(2)} \bar{w}\right)\right], \\
& B_{56}=2 \hat{D}^{(2)}-\bar{v} \hat{D}-(\hat{D} \bar{v})+\mathrm{i} \omega R, \\
& B_{57}=-\mathrm{i} R \bar{w} \\
& B_{58}=-1 .
\end{aligned}
$$

Equation (11) is then solved for the determination of the eigenvalue $\beta$ and the corresponding eigenvectors $\tilde{u}$ and $\tilde{v}$, using standard library software (NAG, 1992). The solution approach is similar, in spirit if not in detail, to that originally proposed by Bridges and Morris (1984). They also used Chebyshev polynomials as basis functions in view of their superior accuracy properties; significantly, though, the present method employs collocation, in our view more straightforward to implement than the tau approach of Bridges and Morris. ${ }^{4}$ Further, the present physical application, unlike that of Bridges and Morris, is on a semi-infinite integration domain and, as such, some form of combination of mapping with domain truncation is required. The interested reader is referred to Theofilis et al. (1994) for implementational details as well as validation against classical test-cases.

The choice of spectral collocation for the discretization of the problem may be defended on

\footnotetext{
${ }^{4}$ Collocation has gained ground over more traditional spectral methods due to the wide availability of efficient (library) software, based on the $\mathrm{QZ}$ algorithm, which appeared at about the time of the work of Bridges and Morris.
} 
grounds of the optimal accuracy offered by Chebyshev polynomials. Accuracy is, of course, the prime consideration of any stability calculation. On the negative side of a spectral approach its cost may be quoted, a consequence of the dense matrices utilized, as opposed to the banded matrices used by a finite-difference method. However, we adhere to the method presented for a number of reasons. First, the (library) eigenvalue solver is based on the QZ algorithm (Wilkinson, 1965) which destroys the bandedness of the matrices involved. Second, the cost per calculation was felt to be acceptable, typically $30 \mathrm{~s}$ on a Cray Y/M-P and less than an order-of-magnitude higher on a Convex C240. An essential ingredient of our approach is its being a global search, capable of delivering the full eigenvalue spectrum. This is unlike the local approach in which an estimated eigenvalue yielded by a coarse global search is purified. In this latter approach information on the spectrum is harder, if at all possible, to obtain. A global search based on second-order finite-differences was ruled out on grounds of the higher cost expected for results of comparable accuracy to those obtained using collocation. Perhaps a viable alternative discretization for the global problem could be a fourth-order compact finite-difference scheme; such a possibility was not exploited in the present solution since it has already been applied in the temporal approach of Hall et al. (1984) and it is of interest to compare the relative performance of the numerical methods.

\section{Results}

In validating the solution method, it is convenient (and physicaly meaningful) to focus on a neutral solution obtained by the temporal approach. In this case, of course, a temporal and a spatial linear analysis yield identical results. Hall et al. (1984) presented Branch II neutral eigenvalues for the zero suction case at $R=800$. Their converged result, obtained with a different numerical approach, is presented alongside our grid refinement history in Table 1 . In obtaining our result a frequency identical to that found by Hall et al. to correspond to neutral waves, $\omega=0.1270977$, was utilized. It may be noticed that our approach, being spectral, utilizes roughly one-third of the nodes used by Hall et al., who used compact finite differences, for a result of comparable accuracy. The neutral eigenfunctions pertinent to this case are presented in Figure 3. ${ }^{5}$

Results are presented next, first for the no-suction case. The temporal mode of immediate importance, from a physical point of view, has been discussed by Hall et al. (1984). This mode, hereafter termed Mode I, may be related to the experimentally observed instability waves (Poll, 1979). The spatial equivalent of Mode $I$ is presented in Figure 4 as growth rate dependence on frequency.

The neutral loop pertinent to the zero suction case is presented in Figure 5(a) in Reynolds number-frequency space. A spatial and a temporal analysis yield, as already mentioned, identical neutral loops. The neutral loop pertinent to the problem (1)-(2) was first obtained in the temporal

Table 1. Grid refinement history in the numerical solution of (3) at Branch II neutral point. Temporal result obtained by $N=160$ points and compact finite differences (Hall et al, 1984): $\beta=0.3384638, \beta c_{\mathrm{r}}=$ 0.1270977 .

\begin{tabular}{ccr}
\hline$N$ & $\beta_{\mathrm{r}}$ & \multicolumn{1}{c}{$\beta_{\mathrm{i}}$} \\
\hline 16 & 0.3460860 & -0.0009085 \\
24 & 0.3346552 & 0.0003027 \\
32 & 0.3385368 & 0.0004374 \\
48 & 0.3384651 & 0.0000012 \\
64 & 0.3384631 & 0.0000005 \\
\hline
\end{tabular}

\footnotetext{
${ }^{5}$ No cosmetic postprocessing of the spectral results presented in Figure 3 has been applied; as a consequence of the clustering of nodes near the wall, the streamwise perturbation appears underresolved. This is an effect of the graphical software; the accuracy of the eigenvectors obtained is comparable with that of the eigenvalues presented in Table 1.
} 


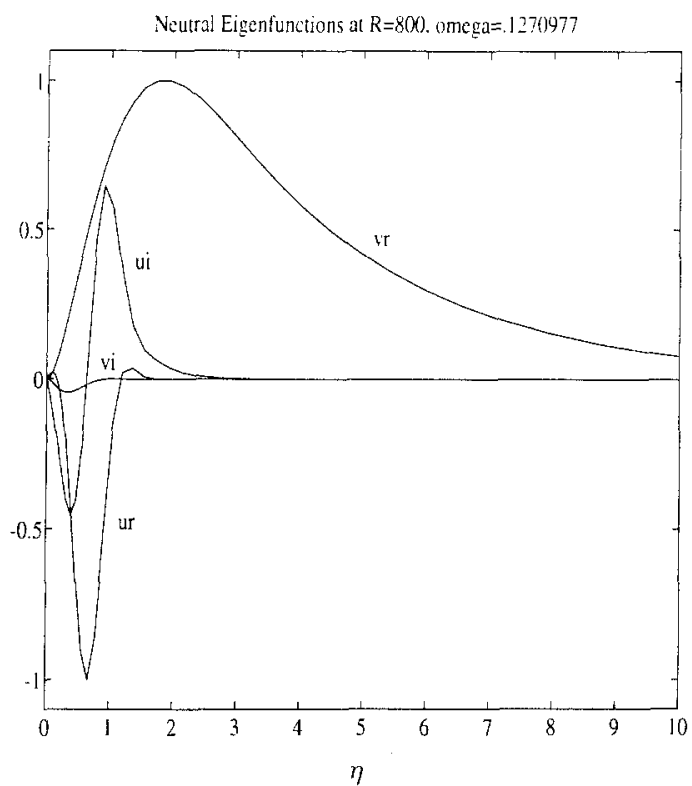

Figure 3. Neutral eigenfunctions at $R=800, \omega=0.1270977$ computed growth rate: $\beta=(0.3384631,0.0000005)$; number of spectral nodes utilized: 64 .

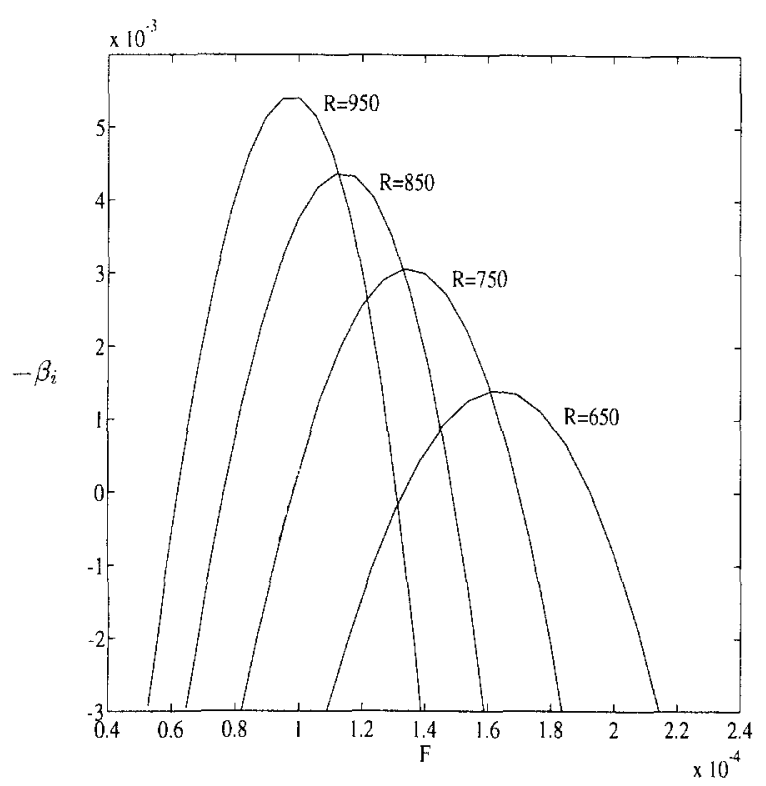

Figure 4. Spatial growth rate $-\beta_{\mathrm{i}}$ against scaled frequency $F=\omega / R$.

work of Hall et al. (1984) and, hence, this result is not discussed further. The frequency that corresponds to maximum amplification at a given Reynolds number may be calculated; this result may be found in tabular form in Table 2. Graphically, the line of maximum amplification rate, the outline of the neutral loop, and the experimental results of Pfenninger and Bacon (1969) and Poll (1979) are presented in momentum thickness Reynolds number ${ }^{6}$-frequency space in Figure 5 (b).

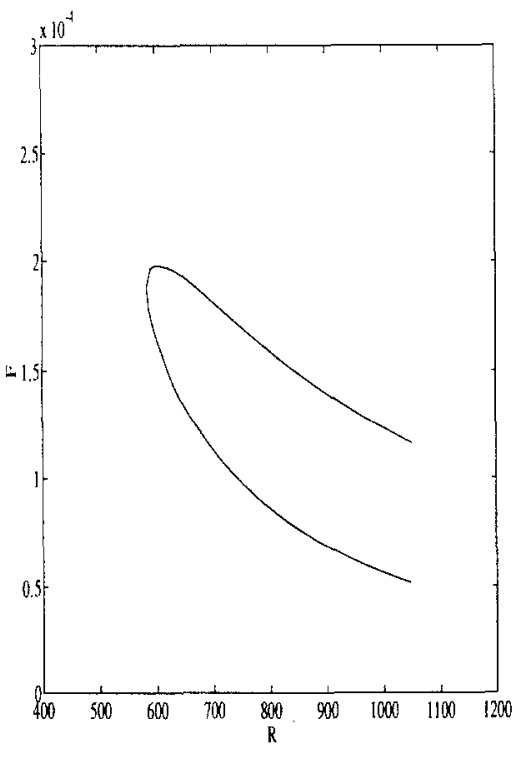

(a)

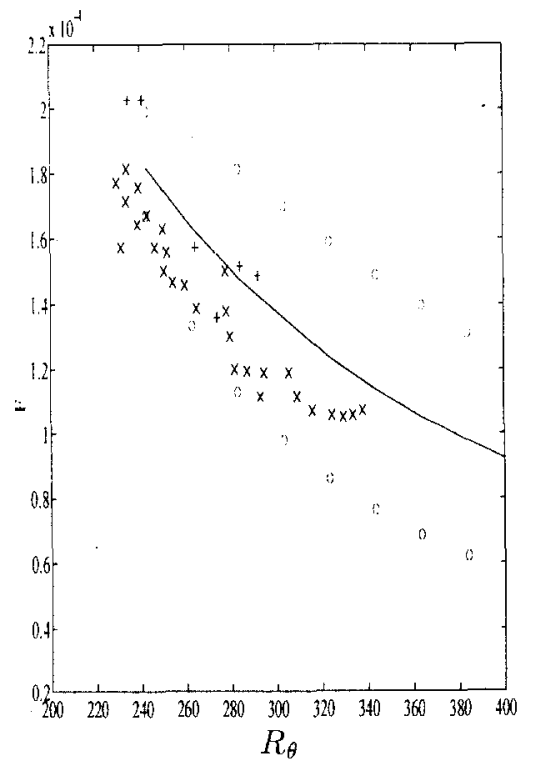

(b)

Figure 5. (a) Linear neutral loop; result identical to the temporal work of Hall et al. (1984). (b) Maximum spatial amplification rate against momentum thickness Reynolds number; superimposed are the boundaries of the neutral loop ( 0 ) and experimental results of Pfenninger and Bacon (1969) $(x)$ and Poll $(1979)(+)$.

\footnotetext{
${ }^{6}$ In the incompressible limit, with no suction applied, $R_{\theta} \approx 0.404 R$.
} 
Table 2. Frequency $F=\omega / R$ and spatial amplification rate $\beta_{i}$ of the maximally amplified wave as a function of Reynolds number $R$.

\begin{tabular}{rrr}
\hline \multicolumn{1}{c}{$R$} & $F \times 10^{5}$ & $-\beta_{\mathrm{i}} \times 10^{4}$ \\
\hline 600 & 18.17 & 3.79 \\
700 & 14.79 & 22.83 \\
800 & 12.35 & 37.57 \\
900 & 10.52 & 49.29 \\
1000 & 9.09 & 58.80 \\
1100 & 7.97 & 66.66 \\
1200 & 7.06 & 73.24 \\
1300 & 6.32 & 78.82 \\
1400 & 5.69 & 83.58 \\
\hline
\end{tabular}

The location in parameter space of the experimental results is the same as that predicted by the temporal results of Hall et al. (1984). Within experimental error, growing waves between Branch I and the line of maximum amplification are found. Growing waves have also been observed, experimentally, below the linear critical Reynolds number; at higher Reynolds numbers instability waves tend to be observed nearer the maximum amplification rate curve. The discrepancy near the tip of the neutral loop is still a matter of controversy; while theory supported by the computation of Hall and Malik (1986) suggested nonlinear subcritical bifurcations, later computations by Spalart (1988), Jiménez et al. (1990), and Theofilis (1994) failed to confirm the existence of numerical solutions corresponding to growing waves subcritical in Reynolds number. The formally excluded three-dimensionality, a consequence of the Görtler-Hämmerlin assumption, further obscures the relation between linear theory results and measurements. In our view DNS alone is capable of discerning between the mechanisms at play unaccounted for by linear analyses, namely, three-dimensionality and nonlinearity.

Considerations regarding the relevance of linear theory to experiments aside, we present in Figure 6 a new mode that the global procedure yields which was not discussed by Hall et al. (1984). As a matter of fact this mode possesses a double structure but, in view of the character of the two branches being identical, we prefer referring to it as a single mode. This is a linearly stable mode, akin to that observed by Bridges and Morris (1984) in the numerical solution of the classical Orr-Sommerfeld

Figure 6. Spatial growth rate $-\beta_{\mathrm{i}}$ against frequency $F=\omega / R$ at $R=650$.

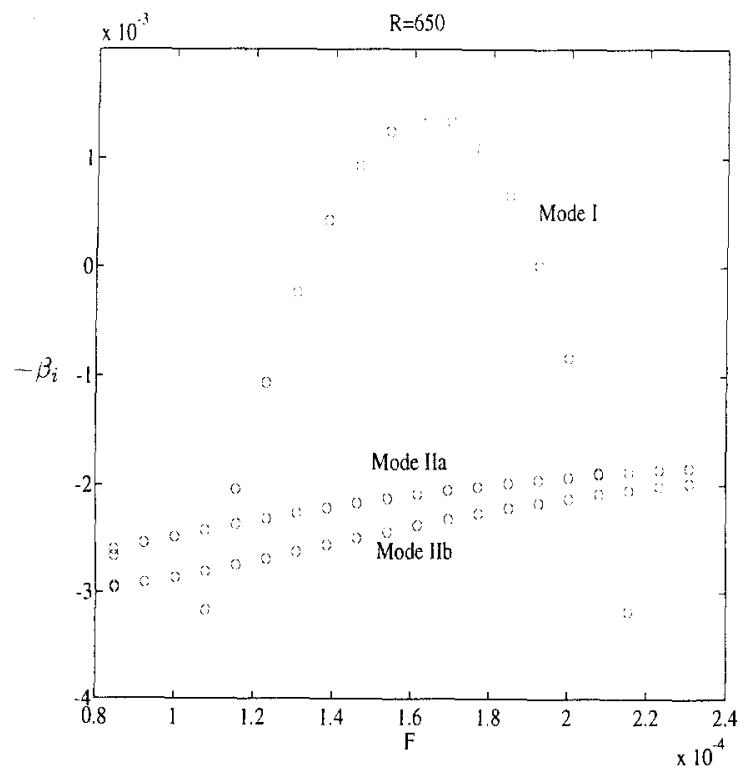




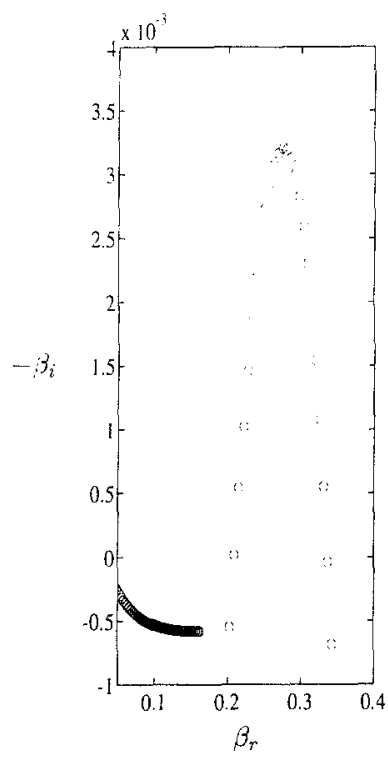

(a)

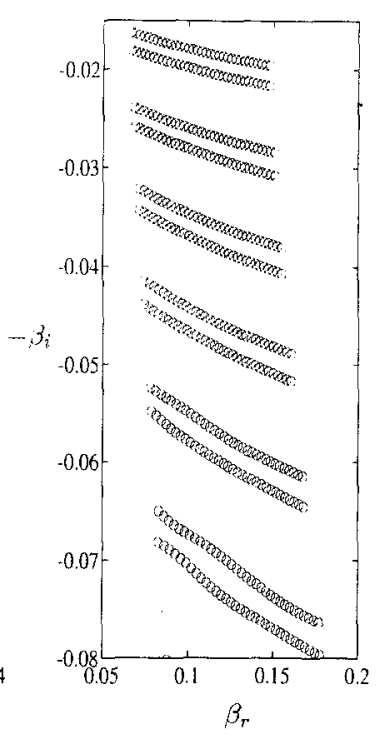

(b)

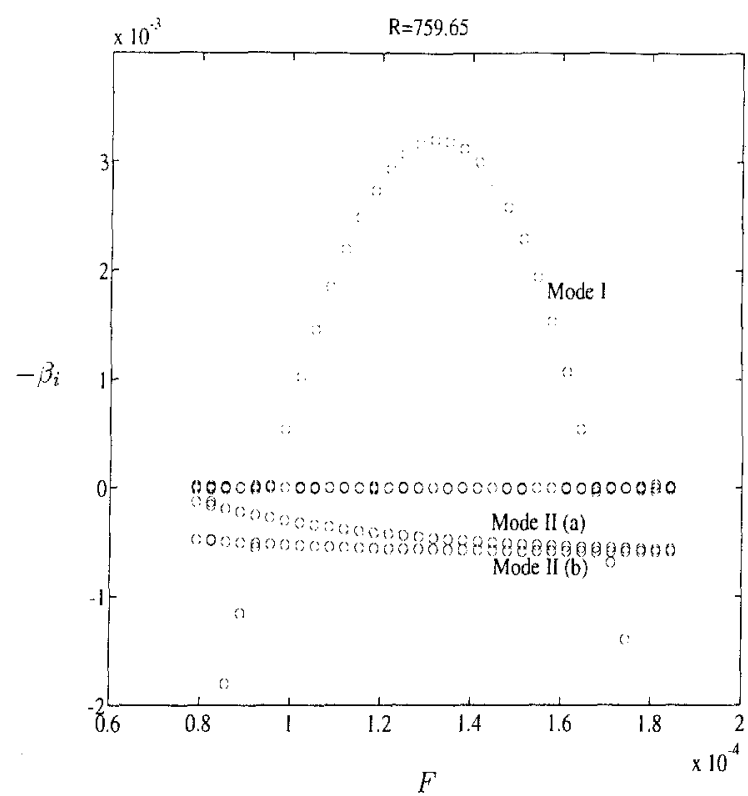

Figure 7. Spatial growth rate $-\beta_{\mathrm{i}}$ against wave number $\beta_{\mathrm{r}}$ at $R=759.65$. Modes $I$ and II are shown (a), as well as very stable pairs of discrete modes (b).

Figure 8. Spatial growth rate $-\beta_{\mathrm{i}}$ against frequency $F=\omega / R$ at $R=759.65$.

equation. ${ }^{7}$ As in the case of the Orr-Sommerfeld modes, there are (stable) frequency regimes in which Mode II dominates over Mode I. It may be argued that Mode II being stable, it has little to do with the transition process. In our view, however, such a definite statement may be made only after the effect of factors such as compressibility on the respective growth rates have been assessed.

Further, by examining the full spectrum of eigenvalues at a given Reynolds number we observe that pairs of stable modes whose ever stronger damping makes them increasingly uninteresting from the point of view of transition to turbulence exist. This result is presented in Figure 7; in (b) the strongly damped modes in question are shown, while in (a) the discrete Modes I and II are presented for comparison. Figure 8 depicts the full spectrum result at $R=759.65$ and the relative tendency of the two branches of Mode II to coalesce may be noticed, compared with the result at $R=650$. As a global comment, valid for all the new modes discussed, notice that they represent long waves whose wave numbers, in the frequency regime explored, terminate at about $\beta_{\mathrm{r}} \approx 0.2$; beyond this value only the dominant Mode I is observed.

As has already been mentioned the stringent condition of decay in the freestream may be relaxed, imposing boundedness instead. A family of eigenvectors which do not merge into the undisturbed stream is then obtained as part of the global solution. These eigenvectors are akin to the modes present in the numerical solution of the Orr-Sommerfeld equation under the condition of boundedness (Grosch and Salwen, 1978); they are depicted in Figure 8 as the line of modes almost aligned with the zero-growth axis (and also exist, but have been omitted from the results presented at $R=650$, Figure 6).

These numerical solutions occupy the full range of wave numbers, starting from extremely low values; the corresponding eigenvectors are oscillatory to the outer limit of the integration domain. In Figures 9-11 we present the spatial structure of a typical example of such a wave. In Figure 9 the streamwise perturbation component $\tilde{u}$ of the oscillatory mode is presented alongside that of the maximally amplified wave at the same conditions, $R=759.65, F=1.32 \times 10^{-4}$. It is immediately observed that the two modes have comparable structure insofar as this perturbation quantity is

\footnotetext{
${ }^{7}$ Bridges and Morris used only Chebyshev polynomials of certain parity and hence obtained in their computations only one branch of Mode II.
} 


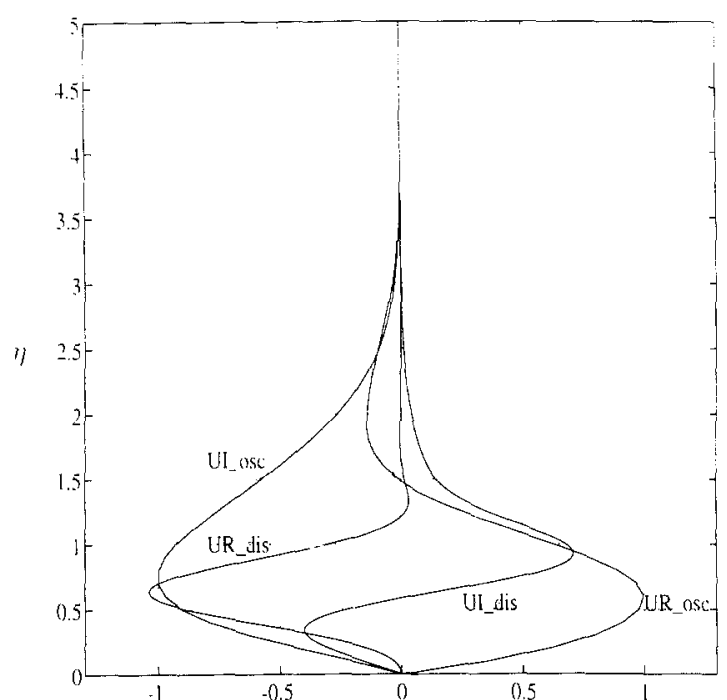

Figure 9. Streamwise perturbation velocity component structure against $\eta$ at $R=759.65$. The real and imaginary parts of the maximally amplified discrete mode and that of an oscillatory mode, denoted by $\mathrm{UR}_{- \text {dis }}$, UI -dis$_{\text {dis }}$ UR $\mathrm{R}_{\text {-osc }}$, and UI $\mathrm{UI}_{- \text {osc }}$, respectively, are shown.

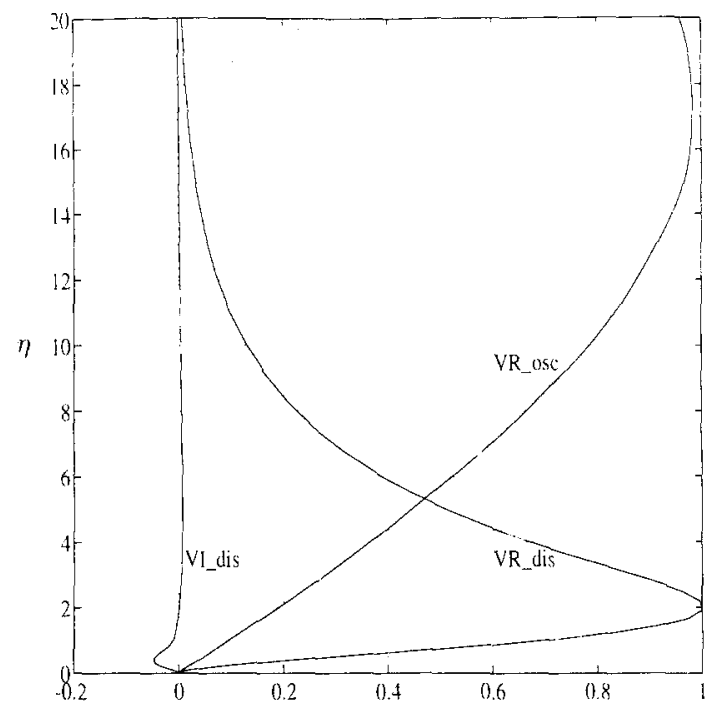

Figure 10. Near-wall structure of normal perturbation velocity component at $R=759.65$. The real and imaginary parts of the maximally amplified (discrete) mode, respectively denoted by $V_{\text {-dis }}$ and $\mathrm{VI}_{\mathrm{dis}}$ and the real part of one oscillatory mode, denoted by VR_osc, are shown.

concerned, $\tilde{u}$ being confined within the boundary layer. However, the normal velocity component of the two waves is fundamentally different, as shown in Figure 10; that of the discrete Mode I exhibits the usual vortical structure with the decay rate discussed earlier, while $\tilde{v}$ of the new mode introduced by the relaxed condition of boundedness corresponds to a sinusoidal wave extending to the outer edge of the computational domain. This latter, oscillatory mode, may be visualized in Figure 11, where the disparity between the length scales associated with the two modes may also be noticed. The period of oscillation (in $\eta$ ) is varied between the different oscillatory modes obtained numerically at a fixed set of parameters. In the present case the result of Figure 11 is that corresponding to the highest frequency; a sequence of discrete modes whose period grows from that presented in Figure 11 to that of a half-sinus over the whole $\eta$ integration range is obtained. As the number of nodes utilized for the solution of the eigenproblem (1)-(2) is increased, this discrete sequence of periods of modes tends to a continuum.

Figure 11. Normal perturbation velocity component structure against $\eta$ of the oscillatory mode of Figure 10. The real and imaginary parts, respectively denoted by $\mathrm{VR}_{- \text {osc }}$ and $\mathrm{VI}_{- \text {osc }}$, are shown.

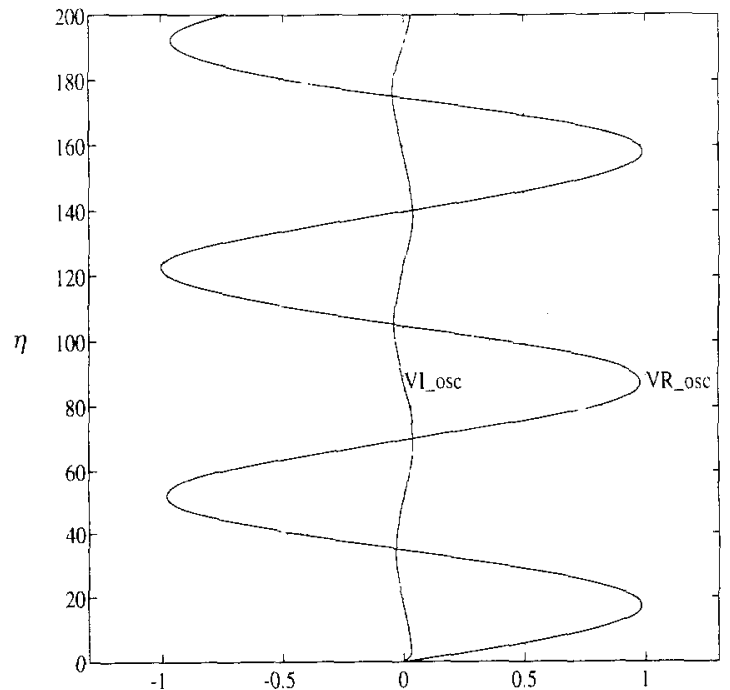


Although the growth rates of the oscillatory modes are comparable with that of the dominant discrete mode, the obvious reservation regarding the relevance of the former modes to the physical problem stems from the fact that the oscillatory modes do not attain the undisturbed freestream value. The structure of the bounded modes in the attachment-line problem and the Orr-Sommerfeld equation being similar, it would be expected that the conjectures regarding the role of freestream vorticity on bypass transition (Grosch and Salwen, 1978) would apply in the present problem, as well. However, in this paper we present the oscillatory modes as solutions of the model system of equations (1)-(2) and leave further discussion regarding their physical interpretation for a later study.

\section{Conclusions}

A spatial analysis of the incompressible attachment-line problem is presented. This work aims at complementing that of Hall et al. (1984) by providing spectrum information inaccessible, numerically, to their local temporal approach. Furthermore, the spatial results obtained should be directly amenable to comparison with experiments without the need for a Gaster transformation. However, this work suffers from the same limitations of the analysis of Hall et al. (1984), namely, the Görtler-Hämmerlin assumption, which may only be defended based on DNS experience (Spalart, 1988).

Within its limitations, the present global spatial analysis has yielded the full spectrum of eigenvalues. Results obtained are:

(a) The existence of a mode, termed Mode II, akin to that present in the Orr-Sommerfeld problem.

(b) The amenability of the continuous spectrum to analysis and its merging into the Orr-Sommerfeld spectrum at large Reynolds numbers.

(c) The appearance in the spectrum of sinusoidal instability modes when the decay condition in the far-field is replaced by one of boundedness.

The solution method for the spatial stability problem may, conceptually, easily be extended to solve the (global) compressible problem (Malik and Beckwith, 1988; Lasseigne and Jackson, 1992), although at a larger computing cost. Such a study would be of interest, however, in order to assess the behavior of the additional (stable) modes presented in this paper; in particular, the relation of Mode II and the Mack modes expected at high Mach numbers, as generalized inflection point criteria (Mack, 1984; Duck, 1990) come into play, would be worthy of exploration.

\section{Acknowledgments}

The author wishes to acknowledge discussions with Professor D.I.A. Poll during the course of the present work. Computations were performed on the Convex C240 of the University of Twente, as well as on the Cray Y-MP/4 of the Stichting Academisch Rekencentrum Amsterdam under NCF Grant $93 / 0138$.

\section{References}

Arnal, D. (1992). Boundary layer transition: prediction, application to drag reduction. AGARD Report 786, p. 5-1.

Boyd, J.P. (1989). Chebyshev and Fourier Spectral Methods. Lecture Notes in Engineering, Vol. 49. Springer-Verlag, Berlin.

Bridges, T.J., and Morris, P.J. (1984). Differential eigenvalue problems in which the parameter appears nonlinearly. $J$. Comput. Phys., 55, 437.

Canuto, C., Hussaini, M.Y., Quarteroni, A., and Zang, T.A. (1993). Spectral Methods in Fluid Dynamics. Springer-Verlag, Berlin.

Duck, P.W. (1990). The inviscid axisymmetric stability of the supersonic flow along a circular cylinder. NASA CR-181816 and J. Fluid Mech., 214, 611.

Gaster, M. (1962). A note on the relation between temporally increasing and spatially increasing disturbances in hydrodynamic stability. J. Fluid Mech., 14, 222.

Görtler, H. (1955). Dreidimensionale Instabilität der ebenen Staupunktströmung gegenüber wirbelartigen Störungen. In 50 Jahre Grenzschichtforschung (H. Görtler and W. Tollmien, eds.), 304. Vieweg, Braunschweig. 
Gottlieb, D., Hussaini, M.Y., and Orszag, S.A. (1984). In Spectral Methods for Partial Differential Equations (R.G. Voigt, D. Gottlieb, and M.Y. Hussaini eds.), p. 1. SIAM, Philadelphia, PA.

Grosch, C.E., and Salwen, H. (1978). The continuous spectrum of the Orr-Sommerfeld equation. Part 1. The spectrum and the eigenfunctions. J. Fluid Mech., 87 (1), 33.

Hall, P., and Malik, M.R. (1986). On the instability of a three-dimensional attachment-line boundary layer: weakly nonlinear theory and a numerical approach. J. Fluid Mech., 163, 257.

Hall, P., and Seddougui, S.O. (1990). Wave interactions in a three-dimensional attachment-line boundary layer. J. Fluid Mech., 217, 367.

Hall, P., Malik, M.R., and Poll, D.I.A. (1984). On the stability of an infinite swept attachment-line boundary layer. Proc. Roy. Soc. London Ser. A, 395, 229.

Hämmerlin, G. (1955). Zur instabilitätstheorie der ebenen Staupunktströmung. In 50 Jahre Grenzschichtforschung (H. Görtler and W. Tollmien, eds.), p. 315. Vieweg Braunschweig.

Herbert, T. (1977). Habilitation, Universität Stuttgart.

Hiemenz, K. (1911). Dingl. Polytechn. J., 326, 321.

Jiménez, J., Martel, C., Agüi, J.C., and Zufiria, J.A. (1990). Direct numerical simulation of transition in the incompressible leading edge boundary layer. Technical Note ETSIA MF-903.

Kleiser, L., and Zang, T.A. (1991). Numerical simulation of transition in wall-bounded shear flows. Annu. Rev. Fluid Mech., 23, 495.

Lasseigne, D.G., and Jackson, T.L. (1992). Stability of a nonorthogonal stagnation flow to three-dimensional disturbances. Theoret. Comput. Fluid Dynamics, 3, 207.

Macaraeg, M.G., Streett, C.L., and Hussaini, M.Y. (1988). A spectral collocation solution to the compressible stability eigenvalue problem. NASA TP-2858.

Mack, L.M. (1984). Boundary layer linear stability theory. AGARD Report 709.

Malik, M.R. (1990). Numerical methods for hypersonic boundary layer stability. J. Comput. Phys., 86, 376.

Malik, M.R., and Beckwith, I.E. (1988). Stability of a supersonic boundary layer along a swept leading edge. AGARD CP-438, p. 3-1.

NAG (1992). Mark 15. Numerical Algorithms Group.

Pfenninger, W., and Bacon, J.W. (1969). Amplified laminar boundary layer oscillations and transition at the front of a $45^{\circ}$ swept flat-nosed wing with and without boundary layer suction. In Viscous Drag Reduction (C.S. Wells, ed.), p. 85. Plenum, New York.

Poll, D.I.A. (1978). College of Aeronautics Report 7805.

Poll, D.I.A. (1979). Transition in the infinite swept attachment line boundary layer. Aeronaut. Quart., 30, 607.

Rosenhead, L. (1963). Laminar Boundary Layers. Oxford University Press, Oxford.

Schlichting, H. (1979). Boundary Layer Theory. McGraw-Hill, New York.

Spalart, P.R. (1988). Direct numerical study of leading-edge contamination. AGARD CP-438, p. 5-1.

Theofilis, V. (1993). Numerical experiments on the stability of leading edge boundary layer flow: a two-dimensional study. Internat. J. Numer. Methods Fluids, 16, 153.

Theofilis, V. (1994). On subcritical instability of the attachment line boundary layer. AGARD 74th Fluid Dynamics Panel meeting; Paper No. 33; also submitted to J. Fluid Mech.

Theofilis, V., Dijkstra, D., and Zandbergen, P.J. (1994). Stability of the Veldman boundary layers. Proc. 14th Internat. Conf. on Numerical Methods in Fluid Dynamics, Bangalore, India; also submitted to Comput. \& Fluids.

Thumm, A. (1991). Dissertation, Universität Stuttgart.

Wilkinson, J.H. (1965). The Algebraic Eigenvalue Problem. Clarendon Press, Oxford. 